\title{
Obituary
}

\section{Andrée Tabouret-Keller: la défense du bilinguisme}

\author{
Josiane Boutet \\ Professeure émérite à l'université Paris-Sorbonne, co-directrice de la revue \\ Langage et société, Université Sorbonne-Paris, Paris, France \\ boutet@msh-paris.fr
}

Le 20 septembre 2020, Andrée Tabouret-Keller, membre éminente du Advisory Board de Journal of Language Contact depuis sa création, nous a quittés. Née en 1929 en Alsace, dans cette partie de la France qui, depuis 1870, fut alternativement française et allemande, où les deux langues le français et l'allemand furent successivement officielles, et où le dialecte alsacien était la langue première et vernaculaire de tous les Alsaciens. C'est dire que le bilinguisme fut sa patrie originelle. En 1948, Andrée Tabouret-Keller (désormais ATK) entreprit des études supérieures scientifiques, en français, à l'université de Strasbourg. En 1956 elle soutint son Diplôme d'Etudes Supérieures en psychologie appliquée (mention pédagogie), Contribution à l'étude de l'apprentissage scolaire de la langue française en Alsace. Dans ce qui constitua son premier terrain de recherche, elle compara les résultats scolaires et l'intelligence d'élèves ruraux parlant le dialecte alsacien à ceux d'enfants francophones en Bourgogne, également ruraux : elle ne put établir aucune différence notable entre ces deux groupes. Elle montra que les enfants bilingues, à condition d'employer avec eux des tests non verbaux, sont aussi intelligents que les unilingues. On voit déjà à l'œuvre en 1959 sa défiance envers la mesure scientifique de l'intelligence fondée sur les tests verbaux. Dans ses travaux futurs sur l'histoire des idées (en particulier en 2011), ATK reviendra sur cette mesure dont elle montrera comment elle a historiquement participé à la persistance de l'idée de la nocivité du bilinguisme. On voit aussi dans ce mémoire son intérêt 
pour une approche sociale du langage ainsi que les options méthodologiques qui la guideront à l'avenir : une préférence pour l'enquête de terrain et une méthodologie comparative entre groupes sociaux, corrélée à un rejet de la psychologie expérimentale en laboratoire et de la mesure du bilinguisme. ${ }^{1}$

\section{La Rencontre avec La Sociolinguistique En Émergence}

Recrutée comme chercheur en psychologie en 1956 au Centre national de la recherche scientifique (CNRS), ATK voulut y implanter des études sur le bilinguisme. Ce secteur était peu développé en psychologie, une discipline où le bilinguisme était encore considéré comme nocif pour le jeune enfant : car son cerveau, alors considéré comme limité, était supposé ne pas pouvoir traiter deux langues en même temps. Elle fut cependant soutenue dans son projet par deux éminents savants, le psychologue Henri Wallon (1879-1962) et le linguiste Marcel Cohen, ce dernier se montrant particulièrement attentif à cette question, délaissée dans la recherche française, disait-il. En mai 1957, il lui écrivit un courrier plein d'optimisme « à partir d'octobre j'aurai une convention pour préparer la mise sur pied d'un office d'étude des bilinguismes. » Mais cet office ne verra jamais le jour et ATK continuera de chercher un cadre scientifique satisfaisant pour y étudier le bilinguisme.

Ce fut la lecture de l'ouvrage d'Uriel Weinreich, Languages in Contact (1953), qui lui sera décisive car elle y découvrit enfin un véritable projet d'étude scientifique du bilinguisme, ce qu'elle ne trouvait pas en psychologie. C'est grâce à André Martinet (1908-1999) qu'ATK put faire un séjour d'études chez U. Weinreich et chez Einar Haugen à l'été 1962. Cette même année, lors du $9^{\text {e }}$ Congrès des linguistes à Cambridge du 27 au 31 août, elle entendit pour la première fois le terme Sociolinguistics employé par S.K. Chatterji à propos de la situation linguistique en Inde. Lors de ce Congrès, elle intervint avec une communication basée sur son terrain d'étude du bilinguisme alsacien: « Contribution à l'étude sociologique des bilinguismes » (publiée en 1964). Elle fut ainsi très tôt au contact des chercheurs et des travaux nord-américains qui allaient fonder la sociolinguistique, et l'une des rares Françaises à publier dans de grands ouvrages nord-américains comme : en 1968 dans Language problems of developing nations de J. Fishman, Ferguson et Das Gupta ; en 1972 dans Advances in the Sociology of Language de J. Fishman. De même, elle écrivit régulièrement dans la revue fondée en 1974 par J. Fishman (1926-2015), International Journal of the Sociology of Language.

1 Cet article est une version remaniée de mon article « Andrée Tabouret-Keller, une vie dédiée au bilinguisme et à léducation bilingue », à paraitre en anglais dans Journal of Sociolinguistics. 


\section{Vers une Conception Sociale du Bilinguisme en Alsace}

En 1969 elle soutint sa thèse d'Etat en psychologie à Strasbourg, Le bilinguisme de l'enfant avant six ans. Etude en milieu alsacien. Elle y exposa une conception pluridisciplinaire du bilinguisme (convoquant linguistique, sociologie et psychologie) dont l'étude " pourrait s'étendre des faits proprement linguistiques, ce qui entraîne l'étude structurale du processus de contact et de ses conséquences, aux faits sociologiques qui définissent la situation de contact et sa transformation ainsi qu'aux faits psychologiques qui touchent au locuteur ou au groupe de locuteurs et aux rapports originaux qu'ils entretiennent avec leurs différents langages. » (non publiée, 1969:27) Sa thèse comportait pour une part une étude de cas (l'acquisition du langage d'une petite alsacienne dans une famille ouvrière bilingue) et pour une autre, une enquête originale menée dans 4 écoles maternelles rurales d'Alsace pendant deux années, auprès d'enfants de 3 à 6 ans. Ceux-ci arrivaient unilingues en alsacien à l'école et encore en cours d'acquisition de leur langue maternelle du fait de leur âge. A l'école, ils apprenaient une langue seconde, le français. Selon une démarche de terrain qu'on qualifierait aujourd'hui d'observation ethnographique (pour le moins inusuelle en psychologie), ATK observa les classes, les jeux dans les cours de récréation, prit des notes. Elle enregistra les interactions entre enfants, entre institutrices et enfants, elle les transcrivit, souvent en phonétique. Elle décrivit minutieusement l'ensemble des phénomènes de contact entre ces deux langues. Mais le plus innovant sans doute fut, dans un doctorat de psychologie, la prise en compte d'un ensemble de facteurs proprement sociaux dans la construction du bilinguisme par ces enfants. Pour comprendre comment interagissaient alsacien et français, elle proposa : des facteurs relevant du milieu scolaire (rôle du groupe de pairs, de l'institutrice, des méthodes d'enseignement) ; des facteurs relevant des milieux familiaux, ainsi elle observe très finement le rôle de la mécanisation de l'agriculture qui introduisait dans l'alsacien parlé dans les familles rurales de très nombreux emprunts au français (p. 238); et des facteurs relevant de la variabilité individuelle des enfants.

Même si le terme de « sociolinguistique » n'est pas présent dans sa thèse, ${ }^{2}$ on voit clairement comment la conception d'ATK du bilinguisme excède désormais sa discipline d'origine, la psychologie, et comporte des déterminants proprement sociologiques.

2 Si la bibliographie reste très majoritairement de psychologie, cognition et acquisition du langage, on y trouve aussi des auteurs qui sont en train de construire la sociolinguistique: Cohen pour la France, pour les USA, Ferguson, Fishman, Gumperz, Haugen, Weinreich et Le Page pour l'Angleterre. 


\section{Bélize ou « les Situations Linguistiques Hétérogènes »}

Dans les années 196o, elle s'oriente vers la psychologie clinique et la psychanalyse (qu'elle exercera), ce qui va lui permettre de complexifier sa vision du bilinguisme, en particulier sur la relation entre les identités et les langues. Cette question sera au cœur de la grande enquête sociolinguistique à Bélize (ex Honduras britannique). ${ }^{3}$ C'est à la fin des années 196o que Robert Le Page et elle obtiennent un financement de la fondation Ford pour engager un travail de terrain à Bélize. Cette région d'Amérique centrale la confronte à des situations sociolinguistiques et à des contacts de langues infiniment plus complexes que les bilinguismes européens. Dans une démarche qualifiable aujourd'hui de constructiviste, R. Le Page et ATK (1985) montrent comment, dans un milieu multiculturel et multilingue à dominante créole, les individus et les groupes s'approprient dans leurs interactions des éléments et des règles propres à diverses langues, et les constituent en autant d'« actes d'identités » et d'appartenances aux groupes. Par l'analyse d'un grand nombre de cas, les auteurs proposent la notion de linguistic polysystemic complexes pour rendre compte de situations et de productions linguistiques non prédictibles, créatives qui rendent inopérantes les notions classiques de la linguistique comme la langue, la grammaticalité ou l'acceptabilité. Ils ont aussi avancé un modèle de compréhension du processus de pidginisation/créolisation en termes de projection/focusing/diffusion, tout à fait novateur et maintes fois repris par la suite.

La plupart des sociolinguistes du monde ont lu et fait lire l'ouvrage de R. Le Page et A. Tabouret-Keller, Acts of identity, et en ont été profondément marqués dans leurs parcours intellectuels. Comme le rappelle Peter Trudgill dans un avant-propos à la rééddition de cet ouvrage en 2006, celui-ci reste un incontournable pour toute la sociolinguistique internationale, même si, comme le souligne ATK dans son introduction à cette même réédition, il y eut « une diffusion inégale dans les pays francophones et les pays anglophones, au grand avantage de ces derniers. ${ }^{4} \gg(2006: \mathrm{V}$-VI $)$

3 A la suite de cette enquête, elle intègra l'International Group for the Study of Language Standardisation and the Vernacularization of Literacy que dirigeait R. Le Page (Tabouret-Keller et alii 1997).

4 Deux tentatives de traduction en français, par J.-M. Eloy et par G. Varro, n'ont pas pu aboutir. 


\section{Histoire des Idées}

Toute sa vie ATK fut profondément persuadée que nos connaissances ont une historicité et qu'on se doit de la connaître, à la fois pour ne pas répéter en un flux ininterrompu ce que se fit auparavant, et surtout pour pouvoir repérer dans les idées dites nouvelles les influences d'une histoire ancienne. Elle y consacra plusieurs de ses travaux.

Débusquer dans nos idées contemporaines les « rejetons » du passé, c'est ce qu'elle fit lors du colloque, « Contacts de langue. Quels modèles? », organisé à Nice en 1987 par la revue Langage \& Société. Il y eut de passionnants débats, entre autres celui opposant sur la conception du code-switching Pénélope Gardner-Chloros à Shana Poplack. Josiane Hamers, ${ }^{5}$ qui venait de publier avec Michel Blanc Bilinguisme et bilingualité, exposa « Un modèle socio-psychologique du développement bilingue ». ATK y donna une conférence, elle aussi mémorable, «Contacts de langues: deux modèles du XIX ${ }^{\mathrm{e}}$ siècle et leurs rejetons aujourd'hui » (publiée en 1988). Elle exposa comment dans la deuxième moitié du XIX ${ }^{\mathrm{e}}$ siècle la grammaire comparée a produit deux principales conceptions des langues et du changement linguistique - la langue comme organisme vivant avec l'image de l'arbre généalogique (Stammbaumtheorie), et la conception physique de la propagation des ondes (Wellentheorie); modèles qui excluaient le mélange de langues, les Mischsprachen, le contact de langues, le plurilinguisme. Elle souligna combien le linguiste allemand Hugo Schuchardt (1842-1927), dès la seconde moitié du $19^{\mathrm{e}}$ siècle, fut un grand précurseur de l'idée qu'il n'existe pas de langue pure et que toute langue contient du mélange (dialectes, créoles): « Le mélange de langues n'est pas tant l'exception que la règle [...] il n'existe pas de langue totalement libre de mélange [...] La possibilité du mélange n'a de limites d'aucun côté; elle peut aller au maximum comme au minimum. » (H. Schuchardt 1884, traduit et cité par A. Tabouret-Keller 2011: 13) ATK termina sa communication par une conception de l'histoire des idées que je qualifierais de matérialiste ou de politique: « J'espère avoir montré que ce n'est pas parce qu'au $\mathrm{XIX}^{\mathrm{e}}$ siècle l'on raisonnait en termes de langues originaires, parfaites, et bien entendu écrites, que de telles entités hantent aujourd'hui les manières dont les langues sont pensées par nous-mêmes et nos contemporains mais c'est parce que ces schémas sont ceux-là mêmes qui étayent et confortent de nos jours une des principales voies de l'assujettissement en vigueur dans

5 J. Hamers appartenait au Centre international de recherche sur le bilinguisme (СıRB) fondé par William F. Mackey à l'Université Laval à Québec : c'est à un tel centre qu'Andrée rêvait à la fin des années 195 o. 
les grands Etats industriels de l'Europe qui, en établissant la langue au rang d'une institution, en font un des plus sûrs instruments de leur pouvoir dont elle est aussi le symbole. » (1988:13)

Théoricien du mélange de langues, $\mathrm{H}$. Schuchardt était un linguiste cher à ATK, c'est pourquoi, en 2009, c'est avec enthousiasme qu'elle s'engagea sur un projet d'édition de quelques uns de travaux les plus significatifs de Schuchardt. Un projet, initié et porté par Robert Nicolaï, qui se concrétisa tout d'abord par la publication de l'édition bilingue de Textes théoriques et de réflexion $(1885$ - 1925), puis, après s'être enrichi de la collaboration de Katja Ploog,se poursuivra avec la traduction de Slawo-Deutsches und Slawo-Italienisches (1883), œuvre atypique dans son époque, que Schuchardt avait rédigé en hommage à Franz von Miklosich (1) [Malheureusement, elle n'aura pas eu le plaisir de voir paraître ce dernier ouvrage, actuellement en cours de fabrication chez l'éditeur. ${ }^{6}$ Contribuer à l'histoire des idées linguistiques, ce fut aussi s'intéresser à l'histoire de la nomination des langues : comment, par qui et pourquoi se décide-t-on à catégoriser un ensemble de parlers comme une langue avec un nom qui lui soit associé ? Questions éminemment sociolinguistiques, historiques et de portée politique, auxquelles s'attela ATK avec son ouvrage collectif de 1997 Le nom des langues, tome I. Les enjeux de la nomination des langues. ${ }^{7}$

Son intérêt pour l'histoire des idées linguistiques s'affirme dans son ouvrage de synthèse en 2011, Le bilinguisme en procès, cent ans d'errance (1840-1940). C'est dans ce livre qu'ATK, à la fois revient à ses étonnement et indignation de jeunesse : «Comment est-il possible de concevoir le bilinguisme comme nocif?», et continue à déployer le point de vue politique sur l'histoire des idées qu'elle avait présenté en 1988. Prenant le parti d'une historiographie, elle analyse un ensemble de documents - rapports officiels, documents administratifs, conférences et travaux scientifiques - produits durant un siècle en Angleterre, Allemagne, France et USA, en se posant ces questions : quelles sont les conditions d'émergence de l'affirmation de la nocivité du bilinguisme ? De sa persistance ? Quelle part faire aux conditions sociales et historiques qui marquent ce siècle ? Elle nous démontre comment les arguments initiaux de la nocivité du bilinguisme ont pu continuer pendant des décennies à prospérer du fait d'un terrain social favorable : «C'est celui des inégalités dans les ressources,

6 2011. En collaboration avec Robert Nicolaï, édition bilingue de : Textes théoriques et de réflexion (1885 - 1925) (CEuvres choisies, tome I) Lambert-Lucas, Limoges.À paraître. En collaboration avec Robert Nicolaï et Katja Ploog, édition de : Slavo-allemand et slavo-italien (1884) (CEuvres choisies, tome II) Lambert-Lucas, Limoges.

7 Cet ouvrage inaugura une série comprenant aujourd'hui quatre publications. 
dans l'accès à un travail rémunéré suffisant ». (2011: 137) Car ce n’est pas le bilinguisme en lui-même qui pose problème, ce sont les conditions sociales et les difficultés que rencontrent les personnes bilingues (guerres, occupations, migrations) : «Alors que les classes dirigeantes sont largement plurilingues, les locuteurs de formes régionales ou dialectales restent en difficulté face aux exigences de l'unité prétendue d'une langue dominante, face aux contraintes de l'accès à l'instruction dans cette même langue alors que l'instruction est donnée comme facteur majeur de progrès pour tous, tant dans les territoires métropolitains que dans les colonies. Dues aux conditions historiques désastreuses de la cohabitation des langues, ces difficultés, patentes, sont interprétées comme un handicap propre au bilinguisme lui-même.» (2011:136-137)

\section{Une Femme de Conviction}

Afin de pouvoir répondre au plus juste à ses interrogations sur le fait du bilinguisme et afin de le constituer en un objet scientifique, ATK eut toute sa vie une quête et un cheminement pluridisciplinaires. Après des études scientifiques qui lui donnèrent le goût de l'observation précise, ce fut la psychologie où elle ne rencontra pas de cadre satisfaisant pour l'étude du bilinguisme. La sociolinguistique lui offrit un tel cadre et le programme qu'elle recherchait pour l'étude des bilinguismes. La psychologie clinique et la psychanalyse orientèrent sa conception de la relation entre les langues et les identités. Enfin l'histoire des idées a nourri l'ensemble de son cheminement scientifique. En 1994, ATK concevait son itinéraire comme la conduisant vers une discipline integrative qu'elle nommait "Anthropology of Language" : « My own evolution is towards an integrative discipline, an Anthropology of Language with new dimensions trying to integrate sociolinguistics and the psychology of language in its broadest sense that is inclusive at least of the social psychology of language and of psychoanalysis.» (1994: 233)

Avec acharnement et une extrême rigueur de pensée, ATK a été le héraut de la diversité des langues, du bi/plurilinguisme et de l'éducation bilingue, luttant contre les théories de la nocivité du bilinguisme; et ce, aussi bien dans ses publications, ses enseignements, ses conférences dans le monde entier que dans son investissement associatif. C'est grâce à sa détermination (certes avec d'autres chercheurs) que nos jeunes collègues trouvent le terrain du bilinguisme apaisé. Limportance des langues maternelles, de leur acquisition et de leur enseignement pour les enfants vivant en contexte bilingue est devenue de plus en plus reconnue et admise (même si personnellement durant les vingt ans que je viens de passer en formation professionnelle des enseignants, j'ai dû 
inlassablement répéter que, oui, il faut laisser les parents d'origine étrangère éduquer leurs enfants dans leur(s) langue(s) maternelles(s) et que, oui, il faut leur raconter des histoires, chanter, parler dans ces langues plutôt que dans un français mal ou peu maitrisé). Plus personne (ou presque) ne croit à la nocivité du bilinguisme pour le jeune enfant. Plus personne ne pense que l'enfant bilingue sera mentalement handicapé puisque son cerveau a deux fois plus de travail à faire que celui d'un monolingue. Plus personne (ou presque) ne pense que du contact des langues naissent des monstres linguistiques: toutes « vérités scientifiques » contre lesquelles ATK aura lutté sa vie durant.

\section{Références}

Le Page R. B. et Tabouret-Keller A. (1985), Acts of identity. Creole-based approaches to language and ethnicity, Cambridge: Cambridge University Press (réédition, 2006, Fernelmont, E.M.E.).

Tabouret-Keller A. (1964), « Contribution à l'étude sociologique des bilinguismes », Proceedings of the Ninth International Congress of Linguists, Cambridge, Mass., August 27-31. En ligne: https://books.google.fr/books?redir_esc=y\&hl=fr\&id= iDPVAAAAMAAJ\&focus=searchwithinvolume\&q=Tabouret+Keller.

Tabouret-Keller A. (1968), "Sociological factors of language maintenance and shift: A Methodological approach based on European and African exemples", dans J.A. Fishman, C.A. Ferguson et J. Das Grupta (dir.), Language problems of developing nations, New York, Wiley, p. 107-118.

Tabouret-Keller A. (1969), Le bilinguisme de l'enfant avant six ans. Etude en milieu alsacien, Doctorat d'Etat, Université de Strasbourg, ronéo, non publié.

Tabouret-Keller A. (1969), « La motivation des emprunts. Un exemple pris sur le vif de l'apparition d'un sabir », La Linguistique 1, p. 25-6o.

Tabouret-Keller A. (1972), "A contribution to the sociological study of language maintenance and language shift", dans J. A. Fishman (dir.), Advances in the Sociology of Language, The Hague, Mouton, Vol. II, p. 365-376.

Tabouret-Keller A. (1975), « Plurilinguisme: revues des travaux français de 1945 à 1973 », La Linguistique 2, p. 122-137.

Tabouret-Keller A. (dir.) (1981), "Regional Languages in France", International Journal of the Sociology of Language 29.

Tabouret-Keller A. (1982), « Entre bilinguisme et diglossie: du malaise des cloisonnements universitaires au malaise social », La Linguistique 18, p. 19-43.

Tabouret-Keller A. (1983), "Code-switching from a psychological point of view", International Journal of the Sociology of Language 39, p. 139-148. 
Tabouret-Keller A. (1984), « De la répétition dans les sciences sociales », International Journal of the Sociology of Language 45, p. 139-151.

Tabouret-Keller A. (dir.) (1985), "Sociolinguistics in France: Current Research in Urban Setting", International Journal of the Sociology of Language 54.

Tabouret-Keller A. (1987), "Questions en vue d'une psychologie clinique du bilinguisme », dans J. Boutet et G. Vermes (dir.), France, pays multilingue, tome 1 , Paris, L'Harmattan, p. 177-19o.

Tabouret-Keller A. (1988), " Contacts de langues: deux modèles du XIXème siècle et leurs rejetons aujourd'hui », Langage \& Société 43, p. 9-22. En ligne: https://www. persee.fr/docAsPDF/lsoc_o181-4095_1988_num_43_1_2999.pdf).

Tabouret-Keller A. (1990), « Continuité et discontinuité de la transmission de l'emploi de l'alsacien dans deux familles alliées », Plurilinguismes 1, p. 1-12.

Tabouret-Keller A. (1994), "From Sociolinguistics to the Anthropology of Language", dans Paulson Ch Bratt. et G.R. Tucker (dir.), The Early Days of Sociolinguistics. Memories and Reflexions, The Summer Institute of Linguistics, p. 225-233.

Tabouret-Keller A. (dir.) (1997), Le nom des langues, tome I. Les enjeux de la nomination des langues, Louvain, Peeters.

Tabouret-Keller A. (2001), « Pour une vision dynamique des situations linguistiques complexes », La Linguistique 37, p. 21-28.

Tabouret-Keller A. (2001), « Traduction de la préface d'André Martinet à Languages in Contact d'Uriel Weinreich », La Linguistique 37, p. 29-32.

Tabouret-Keller A. (2005), «Plurilinguismes. Vers une prise en compte de leur hétérogénéité et de leur complexité », La Linguistique 41, p. 3-6.

Tabouret-Keller A. (2011), Le bilinguisme en procès, cent ans d'errance (1840-1940), Limoges, Lambert-Lucas.

Tabouret-Keller A. et Luckel F. (1981), « La dynamique sociale du changement linguistique : quelques aspects de la situation rurale en Alsace », International Journal of the Sociology of Language 29, p. 51-70.

Tabouret-Keller A. et Luckel F. (1981), « Maintien de l'alsacien et adoption du français: éléments de la situation linguistique en milieu rural en Alsace », Langages 61, p. 39-62.

Tabouret-Keller A., Le Page R., Gardner Chloros P. et Varro G. (dir.) (1997), Vernacular literacy. A Re-evaluation, Oxford, Clarendon Press.

Tabouret-Keller A. et Gadet F. (dir.) (2003), "Sociolinguistics in France: Theoretical Trends at the Turn of the Century", International Journal of the Sociology of Language 16 o. 